\title{
Interprétation des mesures pluviométriques Lois de la pluviosité
}

\author{
Interpretation of pluviometric measurements \\ Rainfall laws
}

\begin{abstract}
Pour l'hydrologue, les indications du pluviomètre ne sont que les éléments de base qui lui serviront à déterminer la quantité d'eau tombéc en un temps donné sur une surface domnée.

L'hydrologue doit donc disposer ses appareils et «exploiter » leurs indications, compte tenu de très nombreux facteurs dont l'influence se trouve résumée et mise en lumière dans les «lois de la pluviosité $\gg$ (lois de pente, d'altitude, d'éloignement) telles (qu'elles peuvent se déduire at la fois de l'expérience et du raisomnement.

For the hydrologist, the data given by the pluviometer are only basic information which will enable him to determine the definite quanlity of rain which fell on a given surface in a given time.

While arranging his pluviometers and making use of the data obtained, the hydrologist mast, therefore, take into account numerous factors the influence of which is described and illustrated in " the rainfall laws" (slope law, altitude law, distance law), such as they may be deducted together from experience and from analyse.
\end{abstract}

\section{I. - INTRODUCTION}

Il est admis couramment qu'un versant exposé au vent pluvieux est plus arrosé que le versant à l'abri de la crête, et d'une façon générale que la quantité de pluie reçue par une surface inclinée diffère de celle qu'aurait reçue la même surface horizontale.

Les observations sont nombreuses et les communications et mémoires abondants. Nombreux aussi sont les modèles de pluviomètres utilisés, car chacun s'ingénie à imaginer des dispositifs susceptibles de corriger les «erreurs》 qu'il attribue à l'appareil voisin.

Mais tout cet ensemble de travaux ne semble pas avoir abouti à une doctrine bien cohérente.
Il arrive parfois même qu'entre les conclusions de divers auteurs subsistent quelques contradictions. Si à l'examen elles se révèlent plus apparentes que réelles, il n'en reste pas moins qu'elles doment à la Pluviométrie une impression de piétinement et d'imprécision.

La pluie est un phénomène banal et observé depuis fort longtemps. C'est peut-être pour cela que l'on néglige trop souvent de préciser exactement ee que l'on veut mesurer, pourquoi on le mesure et comment doit se faire cette mesure.

Ces divers points méritent cependant bien quelque réflexion.

\section{II. - CONSIDERATIONS GÉNÉRALES}

a) Tout d'abord que faut-il mesurer? Lor'squ'il s'agit de précipitations atmosphériques, il ne peut y avoir aucune ambiguïté : météorologiquement parlant, la précipitation est la quantité d'eau météorıque, liquide ou solide, qui traverse une surface horizontale donnée, la section pluviomėtrique. Cette surface est matérialisée par l'ouverture réceptrice horizontale du pluviomètre, et subsidiairement quand il s'agit de précipitations solides, par la surface. horizontale aussi, sur laquelle elles se déposent (MrrcanTON).
Cela élimine tout système de pluviomètres inclinés (Brooks, Hozck et d'autres auteurs), de pluviomètres en boule (Lotssch, Hass), de vectopluviomètres ou stéréo-pluviomètres (PERS).

Cela élimine aussi toute prétendue distinction entre diverses hauteurs de nrecipitafions telles par exemple que la «hauteur des précipitations météorologiques » qui serait la couche d'eau se rassemblant sur une surface horizontale idéale, et la «hauteur des précipitations hyd"ologiques » c'est-à-dire la hauteur mesurée verticalement d'une couche d'eau que l'on imagine 
immobilisée sur une surface inclinée, sans écoulement ni infiltration ou évaporation.

Il paraît en effet un peu artificiel de considérer en une station deux hauteurs de précipitations qui seront évidemment différentes si l'une est mesurée avec un appareil à receptacle horizontal, l'autre avec un réceptacle incliné. En fait, il tombe sur le sol une quantité de pluie bien déterminée, et c'est dans l'interprétation de cette mesure unique que se sépareront les utilisateurs. Le météorologiste et l'hydrologue ne poursuivent pas les mêmes buts; ils ne pourront avoir les mêmes exigences.

b) C'est donc là qu'il convient de préciser le "pourquoi » de leurs mesures pluviométriques.

Le météorologiste considère la pluie comme un élément descriptif du climat qu'il étudie. Il a besoin de savoir combien en une station il tombe d'eau dans l'année, sous quelle forme, solide ou liquide, elle arrive au sol, et quelle en est la répartition mensuelle ou saisonnière. Ces indications pluviométriques jointes à d'autres observations météorologiques (pression, température, insolation, vents, etc.) lui permettront de définir un «climat». Ce qui l'intéresse done c'est, si on veut l'appeler ainsi, la hauteur des précipitations météorologiques, c'est-à-dire le résultat brut donné par un pluviomètre à ouverture horizontale.

Pour l'hydrologue, au contraire, la pluie est plus qu'un facteur descriptif du climat : c'est un élément constitutif du débit des cours d'eau. L'étude du bilan hydrologique d'un bassin versant le conduira à rechercher quelle quantité totale d'eau s'est abattue sur le bassin. Les indications «ponctuelles» d'une ou de plusieurs stations pluviométriques même très rapprochées, ne lui suffisent plus : il devra intégrer tout le long de la surface continue quil constitue son bassin de réception, la somme des hauteurs d'eau recueillies en chacun des points de la surface.

Cette surface n'est généralement pas horizontale. Comme on ne planimètre sur les cartes que sa projection horizontale, il s'introduira dans les calculs un élément nouveau qui est la pente a du terrain et qui permet de passer de la surface planimétrée à la surface réelle.

D'autre part, si l'on excepte les rares jours où l'atmosphère est absolument calme, la pluie ne tombe jamais verticalement : il faudra donc tenir compte aussi de l'inclinaison $\theta$ des trajectoires des gouttes. Ces deux angles $\alpha$ et $\theta$ sont rarement définis avec précision dans les mémoires.

Il ne me paraît pas inutile de le faire iei pour éviter toute confusion. Je pense que l'on voudra bien m'excuser d'insister un peu sur des points qui paraissent élémentaires, mais qui sont cependant à la base de toute interprétation correcte des mesures pluviométriques.

\section{Grandeurs intervenant dans la mesure des précipitations}

\section{a) Terrain horizontal.}

1. Supposons d'abord l'atmosphère calme : la pluie tombera verticalement.

Sans entrer dans le détail de la formation des gouttes d'ailleurs imparfaitement connu, je rappellerai brièvement quelles sont les idées actuelles sur le mécanisme de la pluie.

On admet généralement que dans nos régions tempérées la précipitation d'un nuage est possible lorsqu'il contient à la fois de la vapeur d'eau sursaturée, des gouttelettes en surfusion et des micro-cristaux de glace. Sous l'action de certaines influences que les «fabricants de pluie artificielle » cherchent justement à provoquer, la vapeur sursaturée se condense sur les cristaux de glace; l'atmosphère devenant plus sèche, les gouttelettes se vaporisent et cette vapeur continue à aller se déposer sur les cristaux de glace. Ceux-ci grossissent, augmentent de poids et finissant par vaincre l'action de la pesanteur et celle des courants ascendants, tombent sous forme de flocons de neige. En traversant l'isotherme $0^{\circ}$ ils fondent et continuent leur chute sous forme de gouttes de pluie.

Pour un observateur situé au sol - et c'est notre cas - tout se passe comme si, du nuage représentant un certain volume d'eau précipitable, les gouttes semblaient se détacher du plan horizontal de l'isotherme $0^{\circ}$.

Si $\gamma$ est le nombre de gouttes partant de l'unité de surface inférieure horizontale du nuage et arrivant donc sur Punité de surface du sol, un terrain de superficie $\mathrm{S}$ supposé ici horizontal recevra : $\gamma \mathrm{S}$ gouttes.

2. Supposons maintenant qu'au voisinage du sol il souffle un vent d'intensité et de direction bien définıes. L'influence perturbatrice du vent sera étudiée plus loin dans le détail, mais admettons pour commencer que celui-ci restant cons-

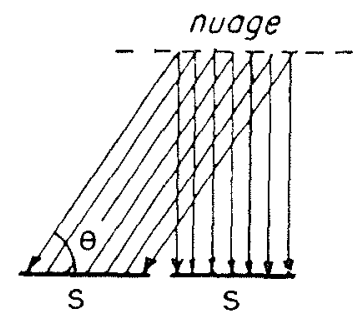

FIG. 1.

tant, ait pour seul effet d'incliner de la même façon toutes les trajectoires des gouttes. Ces trajectoires demeureront parallèles entre elles et atteindront le sol en faisant l'angle 0 . 
Le nombre de gouttes reçu par l'unité de surface reste le même et le terrain en reçoit toujours : $\gamma \mathrm{S}$.

\section{b) Terrain incliné.}

Soit \& la pente du terrain avec l'horizontale, et $O A^{\prime}$ un élément de terrain représenté en coupe, tel que sa projection horizontale $\mathrm{OA}$ soit égale à $S$ (je rappelle que c'est la surface OA que l'on planimètre sur les cartes).

Si la pluie tombe verticalement (atmosphère calme : $\theta=\frac{\pi}{2}$ ), $\mathrm{OA}^{\prime}$ reçoit le même nombre de gouttes que OA. Elles seront seulement un peu
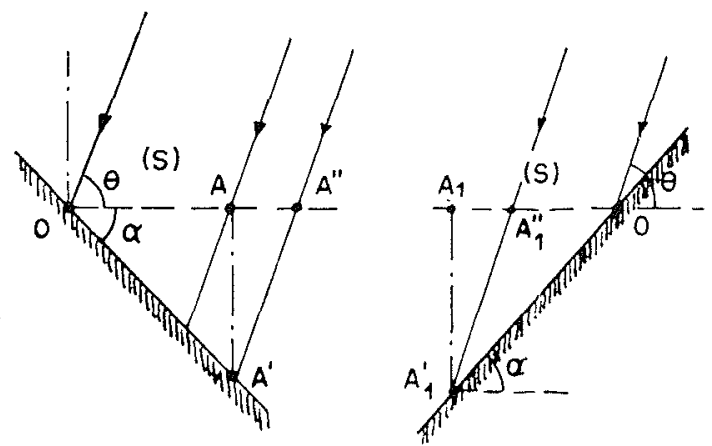

FIg. 2 .

plus «étalées» sur le terrain mais le total de la précipitation reste inchangé.

Si au contraire la pluie tombe obliquement, en faisant un angle 0 avec l'horizontale, il tombera sur le terrain réel $O^{\prime} A^{\prime}$ le même nombre de gouttes qu'il en serait tombé sur la surface horizontale $\mathrm{OA}^{\prime \prime}$.

Un versant attaqué de front par la pluie est donc plus arrosé qu'un terrain de même surface horizontale puisque $\mathrm{OA}^{\prime \prime}>\mathrm{OA}$.

C'est l'inverse pour le versant opposé où $\mathrm{OA}_{1}{ }_{1}<\mathrm{OA}_{1}$.

Un pluviomètre à réceptacle horizontal de section égale à l'unité reçoit donc $\gamma$ gouttes, quantité que l'on exprime d'ailleurs en hauteur d'eau $h$. Ce chiffre suffit au climatologiste. I'hydrologue qui voudra connaitre la quantité totale d'eau reçue par un terrain dont il a planimétré la surface horizontale $S$ devra multiplier la hauteur d'eau mesurée $h$ par $\mathrm{S}$ d'abord et par le rapport $\frac{O^{\prime \prime}}{O A}=R$. Ce rapport représente le taux d'augmentation (ou de diminution dans le cas de la contrepente) des précipitations recues par un terrain de pente a lorsque la pluie tombe obliquement sous l'angle 0 .

Un calcul de géométrie élémentaire montre que :

$$
\mathrm{R}=\frac{\mathrm{OA}^{\prime \prime}}{\mathrm{OA}}=1 \pm \frac{\operatorname{tg} \alpha}{\operatorname{tg} \theta}
$$

Le signe + correspond aux versants exposés à la pluie, le signe - aux versants abrités. L'abaque ci-après traduit ces résultats.

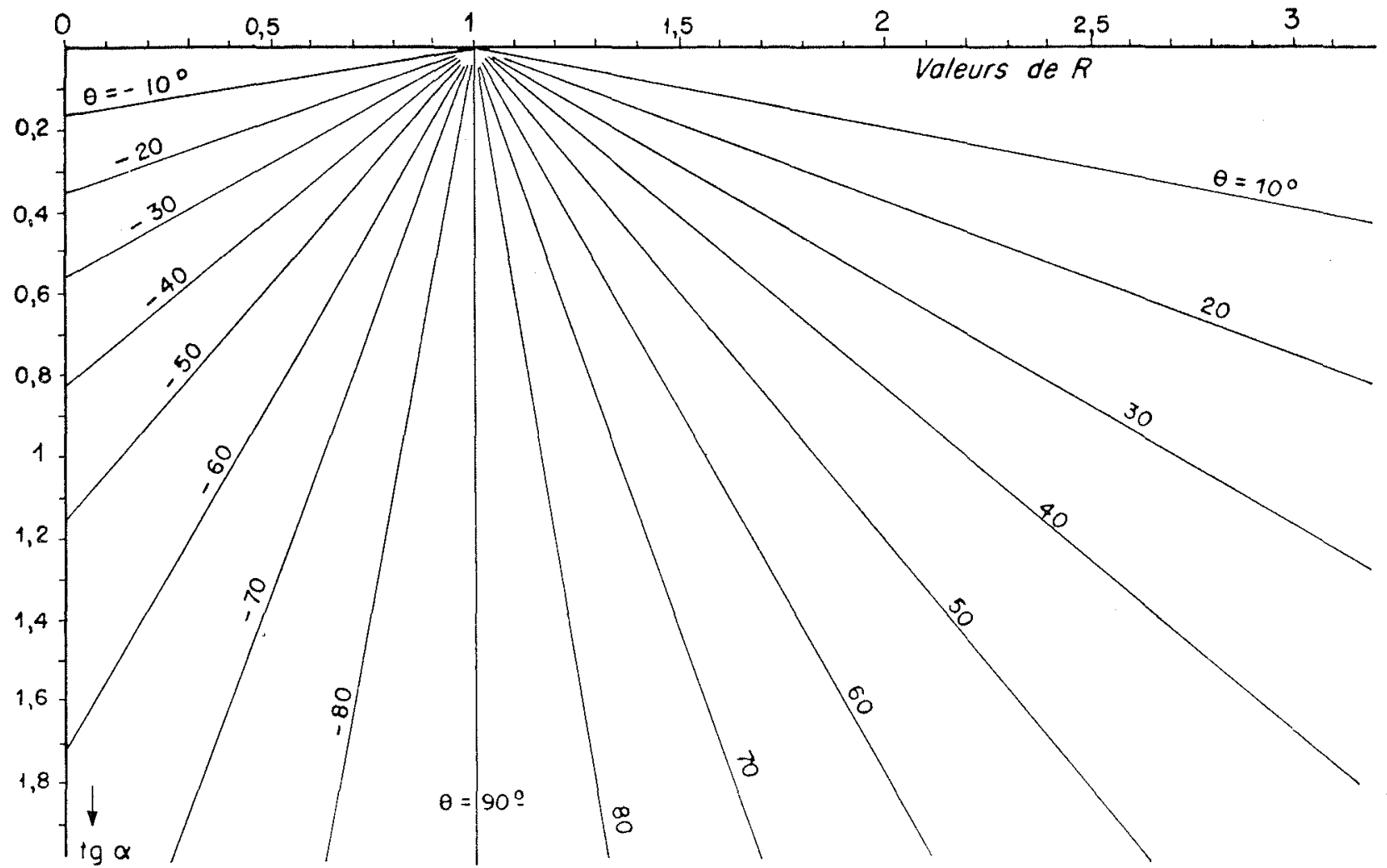

FIG. 3. 


\section{Remarques relatives à l'emploi des pluviomètres inclinés}

a) Certains auteurs préconisent l'usage de pluviomètres inclinés, c'est-à-dire dont la surface réceptrice soit parallèle à la pente du terrain.

Il est indispensable là aussi de préciser comment est exactement réalisé ce parallélisme. On peut en effet (et les deux solutions ont été effectivement expérimentées en Suisse et aux EtatsUnis) :

- soit utiliser un pluviomètre normal à ouverture circulaire o que l'on incline simplement sans autre modification (schéma type b),

- soit couper par un plan oblique parallèle au terrain un tube vertical de section $\sigma$, de façon à obtenir une surface réceptrice de forme elliptique (type c).

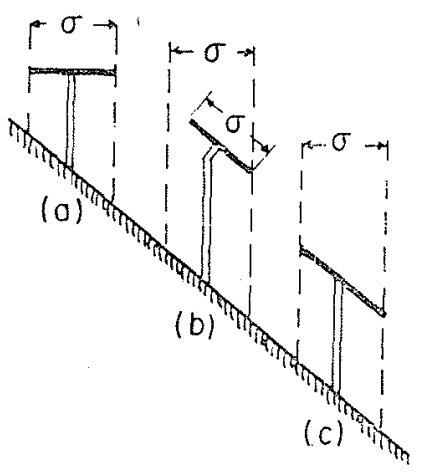

FIg. 4.

On voit aisément que lorsque le pluviomètre horizontal (type a) recueille une hauteur de précipitations $\mathrm{H}$, le deuxième du type $\mathrm{b}$ recevra:

$$
H_{1}=-H \cos x\left(1+\frac{\operatorname{tg} \alpha}{\operatorname{tg} 0}\right)
$$

et le troisième du type $\mathrm{c}$ :

$$
\mathrm{H}_{\varsigma}=\mathrm{H}\left(1+\frac{\operatorname{tg} \alpha}{\operatorname{tg} \theta}\right)
$$

C'est effectivement ce que l'on constate dans tous les essais comparatif's effectués sur des terrains expérimentaux. On ne peut raisonnablement en conchure qu'un modèle est supérieur à l'autre parce qu'il aura donné 20,30 ou $50 \%$ d'eau en plus.
Quant au terrain lui-même, dont la projection horizontale est $\mathrm{S}$, que recueille-t-il?

Dans le premicr cas, ce sera, comme on l'a vu, la quantité :

$$
H \times S \times\left(1+\frac{\operatorname{tg} \alpha}{\operatorname{tg} \theta}\right)
$$

Dans le deuxième cas, ce sera $\mathrm{H}_{\mathrm{b}}$ multiplié par le rapport des surfaces parallèles du réceptacle et du terrain, lequel rapport vaut: $\left(\frac{S}{\cos \alpha}\right)$ sur 1.

Dans le troisième cas, ce sera $\mathrm{H}_{\mathrm{c}}$ multiplié toujours par le rapport des surfaces parallèles qui vaut ici $\left(\frac{\mathrm{S}}{\cos x}\right)$ sur $\left(\frac{1}{\cos \alpha}\right)$ e'est-à-dire S.

Dans les trois cas on retrouve, comme quantité d'eau reçue par le terrain, la même valeur, ce qui est bien évident, car celle-ci ne doit assurément pas dépendre du procédé utilisé pour effectuer les observations.

Cela met en évidence un point qu'en pluviométrie on perd parfois de vue : bien souvent ce que l'on a coutume de considérer comme «erreur de mesure » n'est en fait qu' " erreur d'interprétation de mesures».

b) Mais alors il devrait être indifférent à l'hydrologue d'avoir les valeurs de $H$, ou $H_{b}$ ou $H_{c}$ pourvu qu'il sache de quelle grandeur il s'agit. En principe oui, mais dans la pratique l'installation d'un pluviomètre à ouverture parallèle au terrain restera toujours sujette à caution.

En négligeant, ce qui est normal, les petites inégalités du sol, il esf rare qu'un versant présente une pente uniforme du sommet au fond de la vallée. Le cas figuré par le simple schéma joint est au contraire monnaie courante. Un plu-

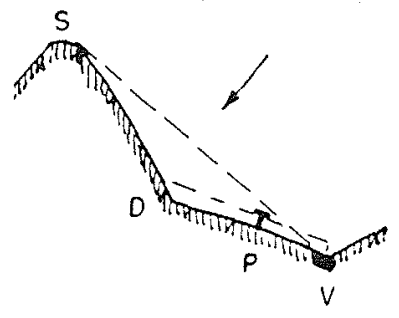

Fic. 5.

viomètre placé en $\mathrm{P}$ devra-t-il avoir son ouverture parallèle à VD ou à VS?

Il est d'autant plus difficile de se prononcer qu'en réalité, ni dans un cas ni dans l'autre, on n'aura une représentation fidèle, de ce qui tombe 
sur l'ensemble des deux éléments constituant le versant total $\mathrm{SV}$.

c) Il faut enfin ajouter que l'introduction dans les réseaux météorologiques existants (où les pluviomètres sont à ouverture horizontale) d'appareils à ouverture inclinée, circulaire ou elliptique, ne peut qu'amener une complication et une source d'erreurs dans les échanges de renseignements entre météorologistes et hydrologues de divers pays.

De ces quelques considérations se dégage ainsi une première conclusion :

Le pluviomètre classique à ouverture horizontale doit demeurer l'appareil universel pour la mesure des précipitations.

\section{Remarque \\ sur l'orientation générale de la pluie}

Lorsqu'on parle de «pente d'un versant», on sous-entend implicitement : angle que fait avec le plan horizontal la ligne de plus grande pente du versant. Et l'on suppose aussi, sans toutefois le préciser, que la pluie, Iorsqu'elle tombe obliquement, attaque toujours le versant perpendiculairement à la crête, c'est-à-dire suivant le plan qui contient la ligne de plus grande pente. Ce n'est cependant là qu'un cas particulier : la plupart du temps le versant est abordé par le travers.

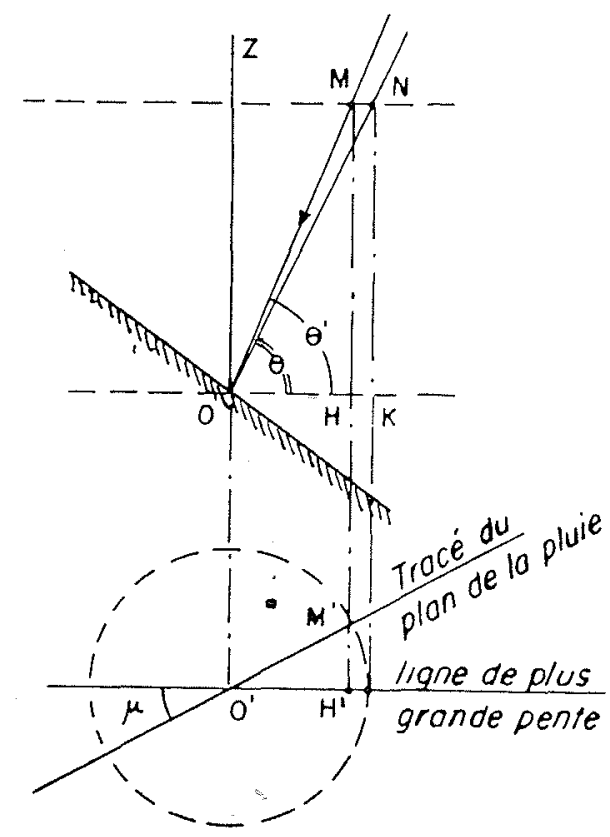

FIG. 6.
Que mesurera alors le pluviomètre?

Soit donc y. l'angle que fait le plan vertical contenant les trajectoires des gouttes (plan de la pluie) avec le plan vertical contenant la ligne de plus grande pente.

La trajectoire réelle d'une goutte est MO (en projection $\mathrm{M}^{\prime} \mathrm{O}^{\prime}$ ). L'angle qu'elle fait avec le plan horizontal est mesuré par $0=\mathrm{NOH}$ rabattement sur le plan de figure.

Mais dans la relation (1) définie plus haut et dans laquelle interviennent l'angle de pente du terrain et l'angle d'inclinaison de la pluie, ce n'est pas l'angle rabattu qui doit être pris en compte mais l'angle $\theta^{\prime}=\mathrm{MOH}$.

$$
\begin{aligned}
& \text { Or on a }: \operatorname{tg} \theta^{\prime}=\frac{\mathrm{MH}}{\mathrm{OH}} \quad \operatorname{tg} \theta=\frac{\mathrm{NK}}{\mathrm{OK}} \\
& \operatorname{tg} \theta^{\prime}=\operatorname{tg} \theta \cdot \frac{\mathrm{OK}}{\mathrm{OH}}=\operatorname{tg} \theta \cdot \frac{O M^{\prime}}{O H^{\prime}}=\operatorname{tg} 0 \cdot \frac{1}{\cos \mu .}
\end{aligned}
$$

Le coefficient $R$ par lequel l'hydrologue devra multiplier la hauteur de précipitations recueillies dans le pluviomètre horizontal devient donc dans le cas le plus général :

$$
R=1+\frac{\operatorname{tg} \alpha}{\operatorname{tg} \theta} \cdot \cos \mu
$$

C'est une relation qui se représente aisément par un abaque à points alignés (voir page suivante).

Tous les cas possibles y figurent:

z varie en effet de 0 (terrain horizontal) à $\frac{\pi}{2}$ (falaise ou mur vertical),

.0: inclinaison de la pluie, de 0 (cas très rares par vents de tempête) à $\frac{\pi}{2}$ (temps calme),

$\mu$ : angle du plan de la pluie avec le plan vertical de la ligne de plus grande pente, varie de 0 à $\frac{\pi}{2}$ (cas où la pluie commençant à attaquer le versant de face tourne jusqu'à devenir parallèle à la crête) puis de $\frac{\pi}{2}$ à $\pi$ (cas du versant «abrité de la pluie $»$ ). 


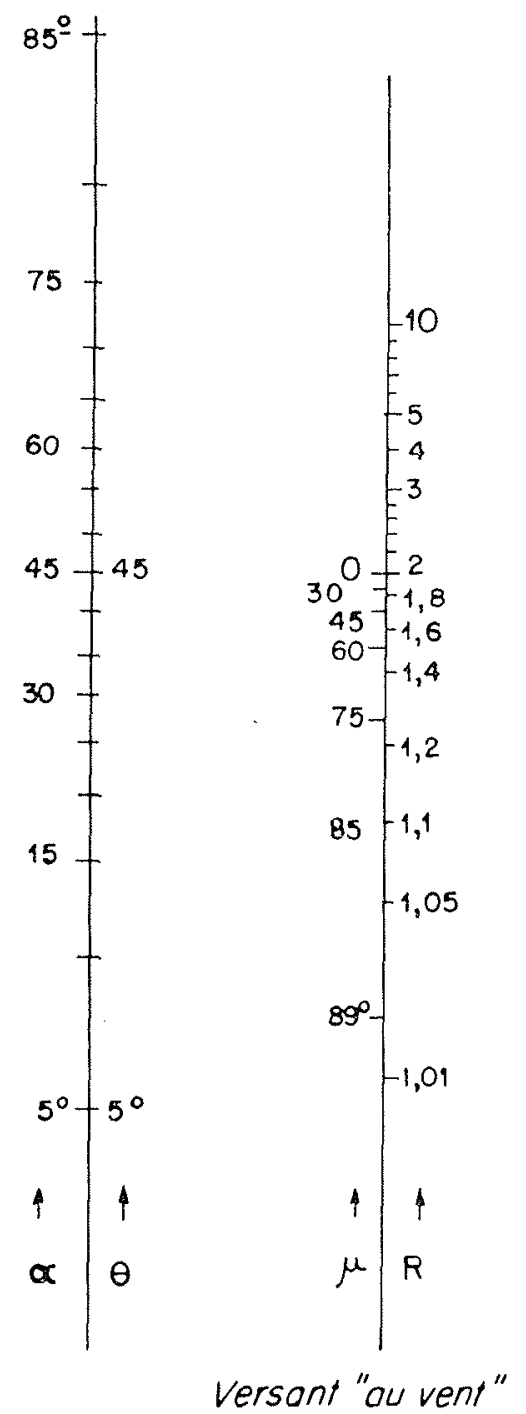

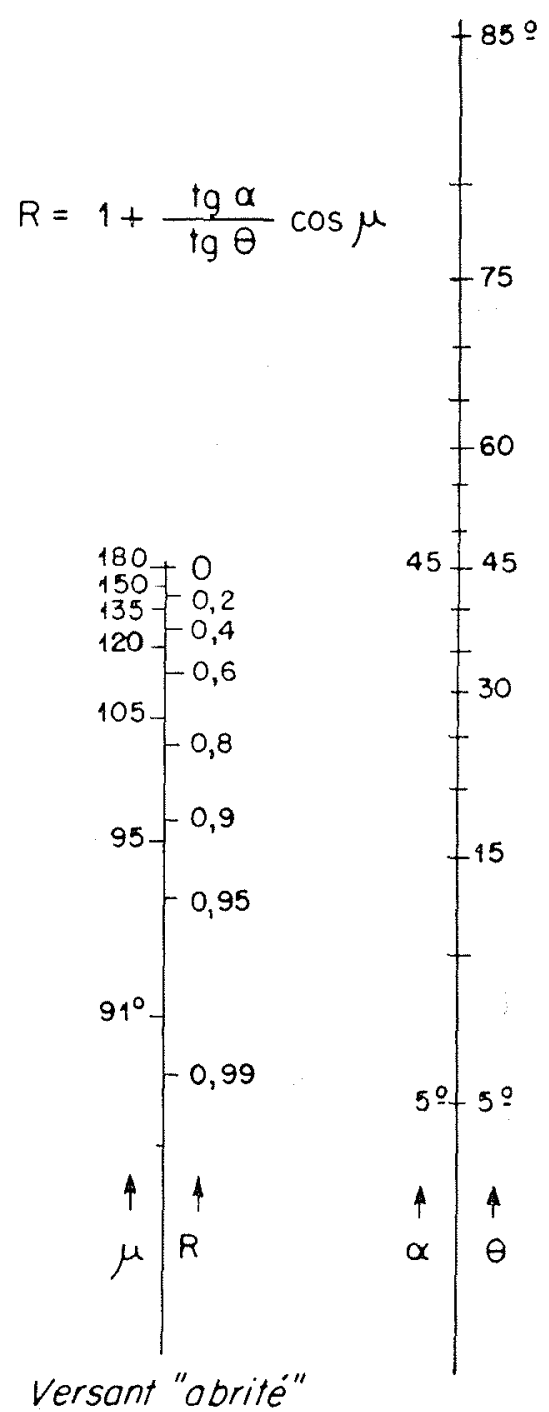

FIG. 7.

Le mode d'emploi de l'abaque est très simple :

On associe d'abord les deux valeurs caractérisant la trajectoire des gouttes, c'est-à-dire $\theta$ et $\mu$. La ligne droite joignant les deux points représentatifs de ces valeurs est prolongée jusqu'à son intersection $X$ avec la charnière non graduée.

On joint ensuite $\mathrm{X}$ au point représentant la valeur de la pente $\alpha$. L'intersection avec l'axe $R$ donne la valeur cherchée.

On utilisera la partie gauche, charnière comprise, pour les versants exposés à la pluie, et la partie droite, charnière comprise, pour les versants abrités. (Ces deux parties, aux graduations identiques, n'ont été séparées que pour éviter des surcharges d'écriture : en fait deux axes et une charnière suffisent ici pour représenter la relation.)
Tout ce qui précède suppose qu'en permanence et simultanément on puisse mesurer les valeurs de et de $\theta$, l'angle $\alpha$ étant fixe. Mais cela ne se justifie que si l'on désire procéder à une analyse fine du phénomène-pluie dans ses rapports avec le débit.

Dans la pratique, on devra se contenter souvent de résultats moyens et consentir pour l'évaluation de $\mu$ et de $\theta$ à quelques approximations.

1. On décomposera d'abord le bassin à étudier en grands versants orientés suivant les huit directions principales N., N.-E., E., S.-E., S., S.-O., O., N.-O. Ayant observé l'orientation moyenne de la direction d'où vient la pluie, les valeurs de $\mu$ seront limitées à des multiples de $\frac{\pi}{4}$ et s'échelonnant de 0 à $\pi$. 
2. En ce qui concerne l'évaluation de 0 , on pourra noter ceci: les gouttes de pluie parcourent avant d'arriver au sol un trajet assez long pour leur permettre d'acquérir leur vitesse limite de chute $V_{1}$. Cette vitesse, qui dépend du rayon $r$ des gouttes, est représentée sur le graphique ci-dešsous (à gauche).

Le champ de répartition des vitesses du vent

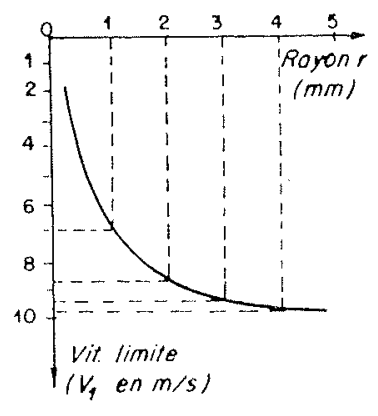

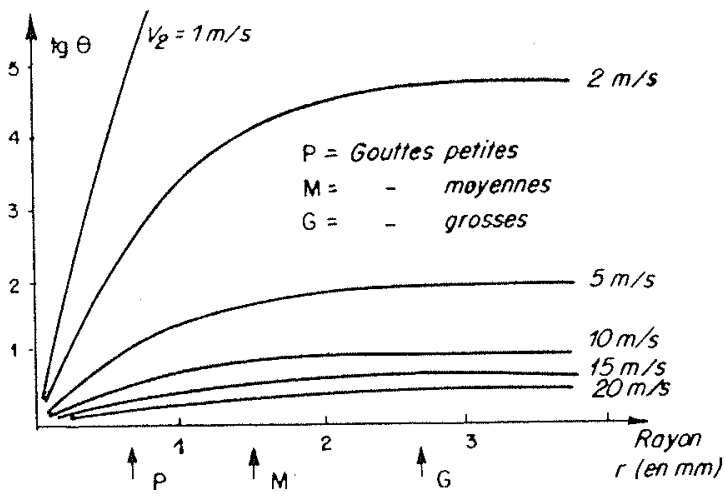

Fifi, 8 : 19. ćtant donné, si l'on suppose, --- ce qui est pratiquement le cas sur un espace restreint - que ce champ est uniforme, l'inertie des gouttes

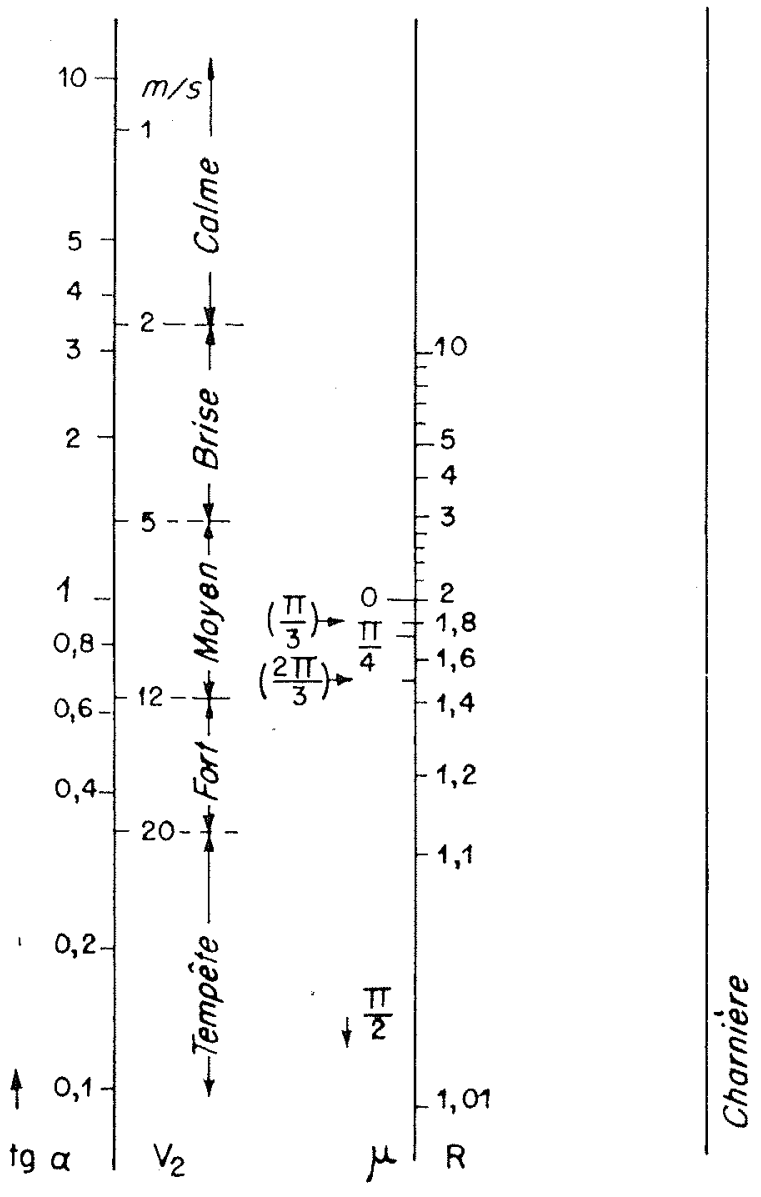

Fig. 10. d'eau n'intervient pas dans leur mouvement de chute; elles prennent alors en chaque point la direction de la vitesse résultante de $V_{1}$ (vitesse limite de chute) et $V_{2}$ (vent).

Cela permet de construire un deuxième graphique (a droite) donnant, en fonction de $V_{2}$, les valeurs de $\operatorname{tg} 0$ pour toutes les dimensions des goultes, petites, moyennes on grosses.

Par suite, il suffira, si l'on admet par exemple que, pour l'ensemble des observations, les gouttes sont moyennes, d'estimer la forec du vent. On le classera en : vent faible (0 à $2 \mathrm{~m} / \mathrm{s}$ ); brise (2 à $5)$; vent moyen (5 à 12 ); fort ( 12 à 20 ), et tempête au-delà.

L'abaque précédent $\mathrm{R}=\mathrm{F}(0, \mu, \alpha)$ peut être alor's remplacé par un abaque «réduit» que l'on utilise de la même facon mais où l'axe $\theta$ est gradué directement en valeurs de $\mathrm{V}_{2}$.

Seule la partie de gauche (versant exposé au rent pluvieux) est représentée ici (fig. 10).

\section{Influence du vent sur les trajectoires des gouttes}

Jusqu'à présent et pour la clarté de l'exposé, j'ai admıs que le champ de répartition des vitesses du vent était uniforme dans tout l'espace, autrement dit que le vent soufflait à vitesse constante sur des pentes sans aspérités; d'où absence de tourbillons, parallélisme des trajec-. toires et constance de l'angle $\theta$ tout le long du versant.

Dans la réalité, les choses sont un peu plus compliquées: le relief terrestre est parsemé d'obstacles qui défléchissent les trajectoires dés filets d'air. 


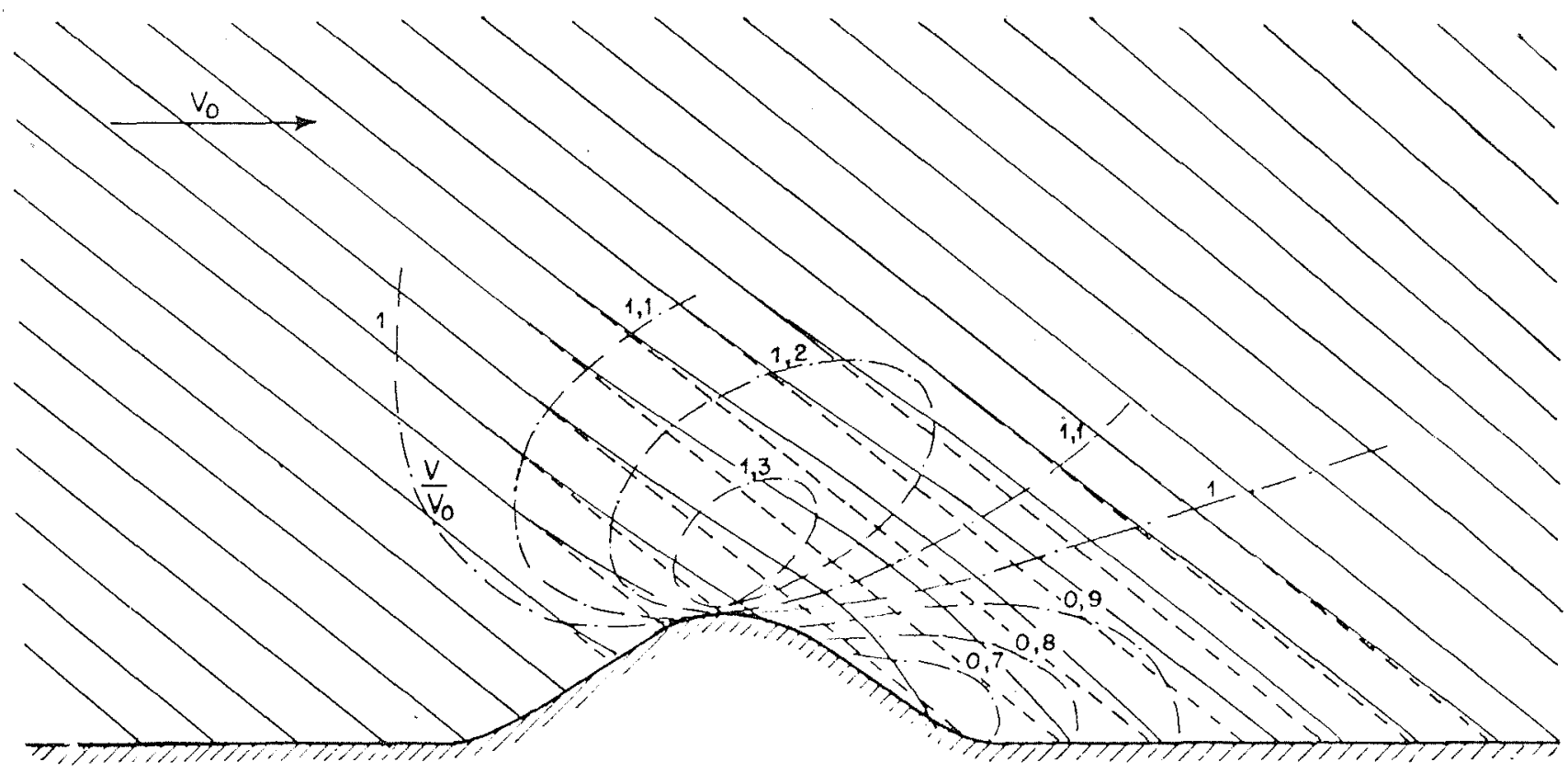

Fra. 11.

Les études théoriques et expérimentales entreprises à ce sujet n'ont fait de véritables progrès que depuis l'essor pris par le vol à voile: elles ne semblent cependant avoir intéressé jusqu'à présent que les aérodynamiciens et les aviateurs.

Dans le domaine de la pluviométrie il a bien été reconnu aussi que le vent pouvait avoir une influence perturbatrice sur la pénétration des gouttes dans l'orifice de l'appareil récepteur; quelques essais ont même été tentés en vue de réaliser des dispositifs «aérodynamiquement neutres à l'égard du vent ». Nipher le premier, puis Bastamoff, Brazier, de Haller, se sont orientés dans cette voie. Mais négligeant d'utiliser les connaissances acquises sur les mouvements généraux de l'atmosphère, ils se sont limités à un domaine d'études trop restreint, le voisinage immédiat du pluviomètre.

En fait, si les déformations des trajectoires restent dues à la même cause généralc : vent buttant sur un obstacle, leur importance est liée aux dimensions de cet obstacle. L'échelle est différente. Il conviendra donc de distinguer les perturbations dues aux formes du terrain de celles qui sont occasionnées par les aspérités locales.

\section{a) Perturbations dues aux formes du terrain.}

Comme je l'ai déjà indiqué, les recherches commencent à être nombreuses, tant sur le plan théorique (Georgin, Pockels, Defant, Taylor, etc.) qu'expérimental (IDrac, Kosmmiener, etc.). Sans entrer dans les détails, il n'est pas inutile de rappeler l'allure générale des résultats que l'on ne peut ignorer dans une étude de la pluviométrie.

Considérons donc le cas simple et courant d'une crète allongée dans le sens perpendiculaire à la direction du vent et figurée par sa coupc sur le schéma ci-dessus. Sans cet obstacle, les trajectoires des gouttes de pluie seraient demeurées parallèles entre elles : droites parallìes si l'on suppose le vent constant dans toute la tranche étudiée, ou courbes parallèles déduites l'une de l'autre par une translation horizontale si l'on considère le cas réel où il existe un gradient du vent avec l'altitude. (Comme on le verra un peu plus loin, seuls seront mesurés les écartements relatifs des trajectoires suivant la direction horizontale, aussi pour la clarté du graphique on a pris le cas où les trajectoires sont, à l'amont de l'obstacle, des droites parallèles.)

Les courbes représentatives des valeurs de $\frac{V}{V_{0}}$ ont été tracées en traits mixtes. ( $V_{0}$ est le vent à l'infini amont, $V$ le vent réel). Cela permet de dessiner les trajectoires réelles des gouttes, en traits pleins : dans la figure on a choisi le «cas moyen», e'est-à-dire: gouttes moyennes (vitesse limite de chute $=8 \mathrm{~m} / \mathrm{s}$ ) et vent moyen $\left(V_{0}=10 \mathrm{~m} / \mathrm{s}\right)$. En pointillé sont figurées les trajectoires théoriques que l'on aurait eues sans la présence de la crête.

L'écartement entre trajectoires réelles successives n'est plus uniforme : il peut varier de $70 \%$ a plus de $150 \%$ de sa valeur normale. Or, cet écartement est inverse de la densité de pluie. L'obstacle a donc, en modifiant la répartition du 
vent, accentué l'efret de la pente sur la densité des précipitations. Entre l'avant et l'arrière d'une crête, il y aura fréquemment des écarts de pluviosité allant du simple au double: le cas est très net sur la figure.

A vrai dire la répartition du champ des vitesses du vent est extrêmement complexe : tout en gardant la même forme générale, le réseau des courbes $\frac{V}{V_{0}}$ se modifie graduellement avec les valeurs de $V_{0}$ et les formes de l'obstacle. On peut tenter de ramener ces formes à des contours géométriques simples que l’on pourra représenter par des formules algébriques: les calculs deviennent vite inextricables. Mais on devra retenir que :

- l'obstacle accentue l'effet géométrique de la pente, éludić dans la première partie de cette note,

- au voisinage de la crète et sur le versant exposé au vent pluvieux l'effet dynamique commence à prendre une valeur relative plus importante: les trajectoires des gouttes s'écartent et l'on constate une diminution de la densité des précipitations. (Cette diminution est d'ailleurs plus ou moins compensée par l'accroissement avec l'altitude et qui est dû à une toute autre cause : on ne mesure en réalité que l'effet résultant),

- les monvements de l'air sont plus réguliers sur les pentes «au vent» que «sous le vent $\geqslant ;$ souns le vent on observe fréquemment la présence de tourbillons stationnaires ou entrainés, ou d'une simple agitation tourbillonnaire. Cette remarque sera utile pour le choix de l'emplacement optinuum d'un pluviomètre.

\section{b) Perturbations alues anx aspérités locales.}

J'appellerai «aspérités locales» les obstacles qui ne sont plus à l'échelle de la montagne; ils peuvent être constitues par des construclions, des rideaux d'arbres, des haies, ete.

Le pluviomètre lui-mème doit être rangé dans cette catégorie : il modifie localement le champ de répartition des vitesses du vent, ec qui peut produire une déflexion supplémentaire des trajectoires des gouttes.

Dans ses études aćrodynamiques, Grorgir a introduit la notion de hauteur d'influence et de distance horizontale d'influence d'un obstacle: cette zone, variable avec l'intensité du vent et les formes du relief, reste de l'ordre de grandeur de l'obstacle lui-mème. Lorsqu'il s'agira d' "aspérités locales», il suffira pour s'affranchir de leur influence de s'en écarter de 3,4 ou 5 fois leur hauteur. C'est d'ailleurs une règle couramment admise.

Reste le pluviomètre lui-même : les perturbations, si légères soient-elles, qu'il provoque dans l'écoulement des filets d'air sont inévitables. Il résulte toutefois aussi bien d'essais en soufflerie (BASTAMOFF) que de calculs théoriques sur les trajectoires des gouttes dans les champs de vitesses normaux, que pour des pluviomètres de dimensions courantes, l'épaisseur des couches d'air déformées reste faible, de l'ordre du décimètre, et que les écarts entre précipitations recueillies par deux appareils, l'un normal, du type Association par exemple, l'autre aérodynamiquement neutre, ne dépassent pas 7 à $8 \%$.

Ce chiffre est d'ailleurs confirmé expérimentalement par des séries d'observations faites à l'Observatoire du parc St-Maur par BrazIER : à 4 mètres d'un pluviomètre ordinaire placé sur un support de $1,05 \mathrm{~m}$ de hauteur, était aménagé un deuxième appareil dont la bague réceptrice affleurait le sol, ce qui éliminait ainsi toutes perturbations dues au pluviomètre et à son support.

Le rapport entre les précipitations recueillies par chacun des appareils a été trouvé égal à 1,05 pour une année entière, avec la répartition saisonnière suivante :

Printemps : 1,06 ; été : 1,03; autornne : 1,03; hiver : 1,08 .

\section{Précision des mesures pluviometriques.}

L'expérience acquise en matière d'aérologie montre que le mouvement de l'air au voisinage du sol n'est pas un écoulement stable, mais qu'il consiste en bouffées et en calmes qu'accompagnent de petits changements de direction. Ces perturbations à période courte sont dues à des tourbillons incrustés dans le courant d'air moyen et répartis dans la masse de l'atmosphère en forme de «cellules» dont la dimension moyenne est de l'ordre d'une trentaine de mètres.

Au cours d'une averse, les gouttes de pluie n'arrivent donc pas sur le pluviometre à la maniere d'un écoulement permanent, mais par rafales irrégulières. Il y a de la sorte entre la quantilé mesurée et celle qui s'est effectivement abattue sur le sol, une marge d'incertitude, que toutes les précautions imaginables ne pourront réduire car elle est due à la nature même du phénoméne que l'on mesure.

La «Division Hydrologie» de l'E.D.F. poursuit actuellement une expérience à ce sujet; sur un terrain expérimental, en un emplacement bien dégagé, ont été disposés en quinconce 16 pluviomètres, écartés d'une vingtaine de mètres l'un de l'autre. Les résultats des premiers mois d'observations font apparaître entre les indications de l'un quelconque d'entre eux et la 
moyenne calculée sur l'ensemble, un écart pouvant atteindre $8 \%$.

C'est l'ordre de grandeur de l'écart constaté entre les résuftats donnés par un pluviomètre ordinaire et ceux d'un pluviomètre «non influencé par le vent $»$. Il apparait illusoire dans ces conditions d'essayer de réaliser un dispositif aérodynamiquement neutre : on ne joue en effet que sur une marge de quelques pour cent que la nature même du phénomène à mesurer empêche de resserrer davantage.

\section{Ecrans protecteurs.}

Pour atténuer l'influence des remous autour des pluviomètres, on a imaginé de les enlourer d'un écran protecteur dont le type est l'écran de Nrpher. Des mesures comparatives ayant montré qu'en général un appareil "protégè » recucillait un peu plus d'eau qu'un appareil nu, on a conclu un peu hâtivenent à l'efficacité du dispositif protecteur.

Or, que se passe-t-il en réalité? Ia déformation des filets d'air produits par un appareil de dimensions courantes (diamètre du pluviomètre Association : $22,5 \mathrm{~cm}$ ) ne s'étend que sur une zone de l'ordre d'une dizaine de centimètres. Un

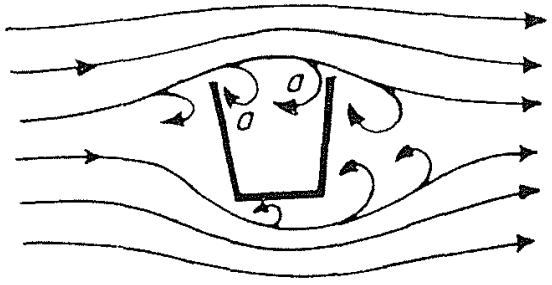

FIG. 12 .

écran du type Nrpuer dont le diamètre est $1,30 \mathrm{~m}$ a pour premier effet d'augmenter les dimensións de l'obstacle. Cela provoque vers l'intérieur du dispositif un appel plus important des filets descendants tels que $a$ a, d'où croissance de la densité de pluie sur la surface réceptrice. Mais il ne s'agit en fait que d'un resserrement, localisé sur l'appareil de mesure, des trajectoires des gouttes d'eau : le résultat ainsi enregistré ne peut donc être considéré comme représentant fidèlement la quantité de pluie tombée sur le terrain voisin autour du pluviomètre. L'expérience directe confirme d'ailleurs parfaitement ces considérations. Des pluviomètres voisins, de dimensions différentes, c'est-à-dire dont les surfaces réceptrices étaient dans les rapports 1 -$16-40-100$ ont donné respectivement des indications dans les rapports 1 - 1,09-1,14 1,20. En dépit même de la marge d'incertitude
(7 à $8 \%$ environ) inhérente à toute mesure pluviometrique, l'influence des dimensions de l'appareil sur la quantité d'eau recueillie reste visible.

Que conclure dès lors sur l'efficacité d'un écran «protecteur»? Resserrant artificiellement sur lui les trajectoires des gouttes, il fausse la valeur de l'échantillon de pluie prélevé, ce qui complique inutilement l'interprétation des résultats. Il doit donc être considéré comme plus nuisible qu'utile, même - et surtout - si les quantités d'eau recueillies sont supérieures à celles qu'aurait donné un pluviomètre non protégé.

\section{Choix de l'emplacement optimum d'un pluviomètre}

Le nombre des stations pluviométriques ne pouvant, pour diverses raisons, etre multiplić à l'infini, le choix des emplacements de mesure revèt une importance particulière.

Une observation pluviometrique n'est en somme qu'un prélevement d'échantillon de pluie: on devara done s'attacher à ce que cet échantillon soit bien « représentatif». Cela conduit à écarter d'emblée tout emplacement et tout dispositif - qui donneront de la grandeur a mesurer des valeurs particulières et difficilement exploitables.

Le dispositif sera, je le rappelle, un pluviometre simple, de dimensions réduites (diamètre de la bague de l'ordre d'une vingtaine de centimètres) à ouverture horizontale et utilisé sans ecran.

Quant au choix de l'emplacement, il déeoule des remarques faites au cours de cette note.

Une comparaison entre mesures pluviométriques et mesures hydrométriques est au reste fort instructive.

Un hydraulicien cherchant à évaluer le débit d'un cours d'eau n'ira pas placer son moulinet trop près des rives ou derrière une pile de pont sous prétexte qu'il se trouve ainsi dans des zones plus «abritées». Et si les circonstances l'empèchent de multiplier ses points de mesure, il choisira sur un profil moyen ceux que lui donnent les valeurs les plus voisines de la vitesse moyenne réelle du courant : points situés au $1 / 5$ et aux $4 / 5$ de la profondeur dont il prendra la moyenne des indications, et à la limite s'il ne peut faire qu'une mesure unique, point situé aux $6 / 10$ de la profondeur en partant de la surface.

C'est à peu près exactement ce qui se passe en pluviométrie : les stafions sont rares, il faut savoir choisir leurs emplacements.

a) On évitera donc en premier lieu les «points singuliers» que sont les zones d'influence d'obstacles tels que rideaux d'arbres, maisons, ete. En particulier, un toit en terrasse, même 
s'il parait bien dégagé, est toujours un mauvais cmplacement. Les météorologistes italiens préconisent de se tenir à une distance de l'obstacle égale à 10 fois au moins sa hauteur. C'est peutêtre faire preuve de beaucoup de prudence, mais cela vaut infiniment mieux que de tomber dans l'excès inverse qui consiste à vouloir rechercher un abri souvent trompeur;

b) S'il n'est possible, et ce sera souvent le cas, de n'installer qu'une seule station sur un versant, il paraît raisonnable de la choisir à mi- pente. On se rapproche ainsi de la régle des $6 / 10$ des hydrauliciens;

c) Le choix du versant lui-même dans la zone à étudier n'est pas indifférent : l'écoulement des filets d'air est moins régulier le long des pentes «sous le vent $»$ où les tourbillons affectent davantage les trajectoires des goultes de pluie que sur les pentes «au vent». On devra donc, pour l'installation des stations pluviométriques, préférer les emplacements situés sur les versants attaqués de front par les vents pluvieux dominants.

\section{III. - APPLICATIONS. - LOIS DE LA PLUVIOSITE}

\section{LOIS DE IA PENTE.}

Elles ont été établies au début de cette note. On peut les résumer ainsi :

a) Efret «géométrique» de la pente. - La quantité d'eau recue par une surface inclinée diffère de celle quauraif reçue la projection horizontale de la surface. Elle est supérieure sur un versant exposé au vent pluvieux, inférieure sur le versant à l'abri de la crête.

Le taux de variation (augmentation ou dimiuution par rapport à la cuantité recue par la surface horizontale) est exprimé par :

$$
R=1+\frac{\operatorname{tg} \alpha}{\operatorname{tg} \theta} \cos \mu
$$

b) Effet «dynamique». - La hauteur d'eau recueillie par un pluviomètre à ouverture horizontale est, à altitude égale, plus grande sur un versant exposé au vent pluvieux que sur le versant abrité. Toutefois sur les pentes au vent, les trajectoires des gouttes commencent à s'écarter l'une de l'autre au voisinage de la crète où l'on observe une diminution de la densité des précipitations.

\section{Lor d'Altitude.}

Une arête montagneuse disposée perpendiculairement à l'axe de marche d'un front pluvieux provoque d'abondantes condensations. D'une manière générale les précipitations augmentent avec l'altitude, sauf cas particuliers qui seront examinés plus loin:

Diverses formules ont été proposées pour exprimer cette loi d'accroissement. Elles ne sont la plupart du temps que la traduction algébrique d'observations expérimentales effectuées en des régions d'étendue limitée et ne peuvent de ce fait être applicuéè a tous les cas. Or, s'il est vain de vouloir établir des lois générales par le seul examen de données globales fournies par les pluviomètres, on peut, en isolant et en étudiant séparément chacune des causes influencant la pluviosité, arriver à dégager d'un phénoméne complexe au premier abord, les quelques lois démentaires qui régissent ses variations.

l'exemple suivant illustre ce procédé de recherche:

Le bassin versant du Var et de la lines dans

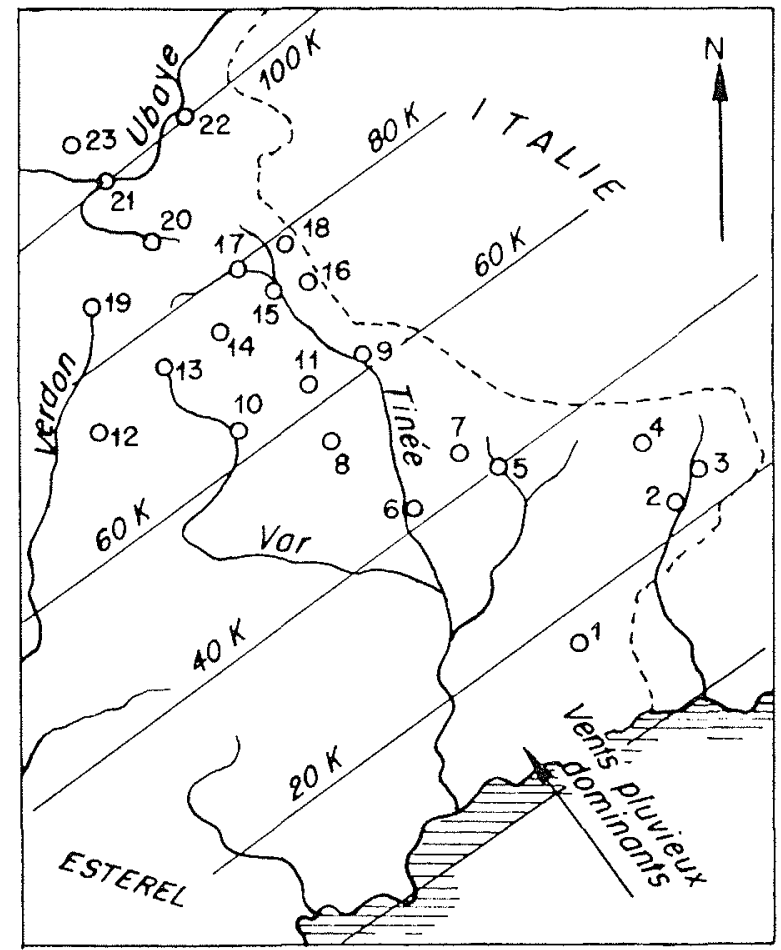

FIG. 13

le sud-est de la France, c'est-à-dire la région limitée par la frontière italienne, le cours supérieur de l'Ubaye, le cours supérieur du Verdon 
et le Massif de l'Esterel, présente une particularité intéressante: dans la presque lolalilé des eas, le vent pluvieux vient du sud-est. Celle région est équipée d'un réseau de 23 stations pluviométriques en service depuis plus de quinze et souvent vingt ans (voir la figure) et qui s'échelonnent entre 220 et 2.730 mètres d'altitude.
La représentation graphique des précipitations annuelles moyennes en chaque station en fonction de l'altitude domne un nuage de points très étalé et peu exploitable. Mais conmaissant la situation géographique exacte de chaque pluviomètre sur des versants exposés ou non aux vents pluvicux du S.-E., on peut, d'après les lois de la
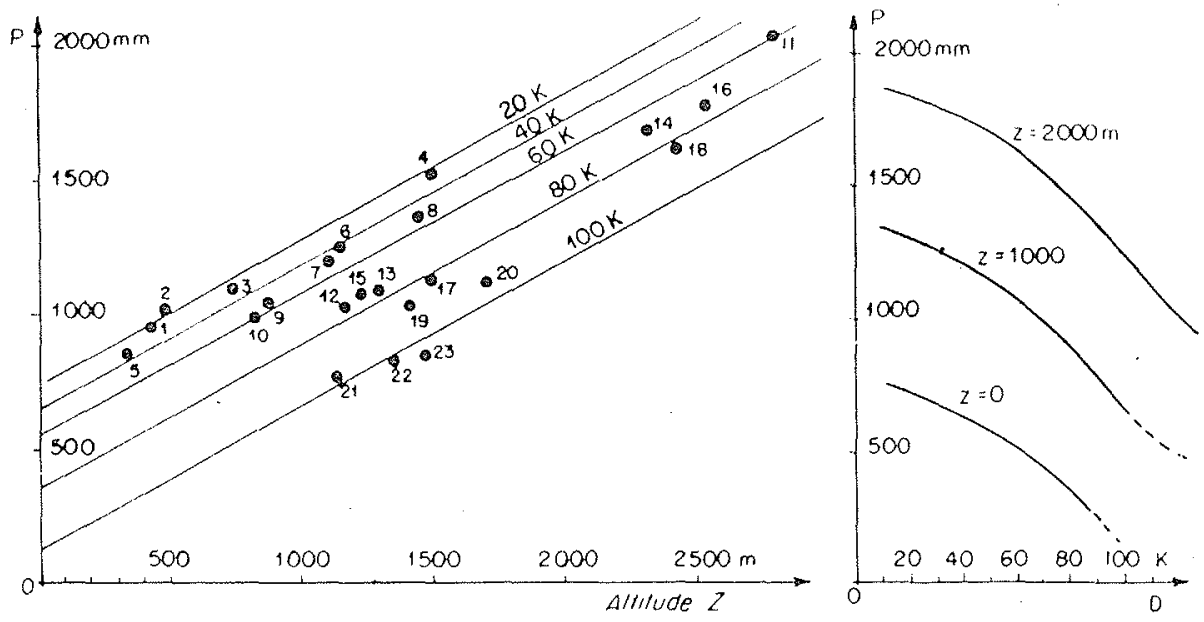

Fla, 14 et 15 .

pente, remplacer les précipitations brutes par des «précipitations réduites 》 d'où est exclue l'influence du facteur abri. Cela donne la représentation figurée ci-dessus (partie gauche).

Le nuage est encore étalé. On constate cependant qu'en reliant entre eux les points figuratifs des stations situées à égale distance de la mer, ces points se rangent très correctement le long d'un réseau de droites parallèles, ce qui permet de formuler la «Ioi èlémentaire d'altitude» suivante :

Pour des stations soumises simultanément à l'influence des mêmes fronts pluvieux, c'est-àdire pour des stations situées à la même distance de la mer, les précipitations croissent linéairement avec l'altitude. Le gradient trouvé ici est : $55 \mathrm{~mm}$ par $100 \mathrm{~m}$ de dénivellée.

Un autre exemple a été étudié : en Italie, dans la région comprise entre Apuana et Viareggio, sur le versant S.-O. de l'Appennin septentrional, on trouve un cas d'application de la loi élémentaire : elle est bien linéaire et le gradient a sensiblement la même valeur.

\section{Cas particulier}

La loi d'altitude ne s’applique que si le massil montagneux abordé par les masses d'air humide en mouvement constitue un front continu. S'il s'agil d'une montagne isolce, une partic du système nuageux pourra, au lieu d'escalader la montagne, la contourner sans se refroidir et se condenser.

On observe dans ce cas une pluviosité inférieure à celle qui devrait correspondre à l'altitude.

\section{Lor D'ÉLOIGNEMENT.}

Si l'on examine maintenant les groupes de stations à la même altitude, ce qui revient à faire dans le graphique précédent $\mathrm{P}=f(Z, D)$ des coupes parallèles à l'axe $\mathrm{O} P$, on obtient le réseau de courbes figurées à droite du graphique : physiquement on peut interpréter leur forme de la façon suivante: les systèmes nuageux s'usant à mesure qu'ils avancent à l'intérieur des continents, les précipitations vont en décroissant mais de façon asymptotique. Mathématiquement, la relation entre la hauteur de pluie $P$ et la distance D à la mer sera de la forme:

$$
\mathrm{P}=\lambda e^{-\mu \mathrm{D}^{2}}
$$

L'exemple suivant en fournit une vérification supplémentaire :

La région choisie est située entre la Garonne 
et les premiers contreforts des Pyrénées. Si l'on ne considire d'une part que 36 stations situces entre 5 et $157 \mathrm{~m}$ d'altitude (pour rendre à peu près négligeable l'influence de l'altitude) et si I'on se limite d'autre part aux précipilations d'automne (en octobre-novembre et décembre les vents pluvieux conservent tous la même direction : O. - S.-O.) on obtient une courbe $\mathrm{P}=f(\mathrm{D})$

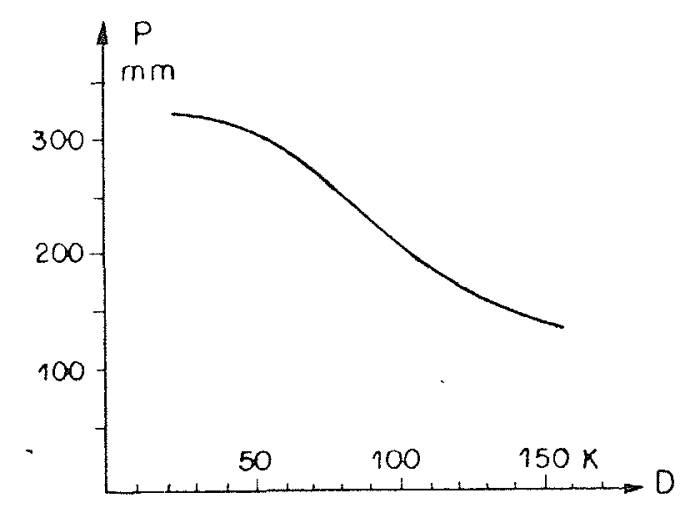

FIG. 16.

dont la forme très caractéristique répond exactement à la relation indiquée ci-dessus.

Cela permet de formuler la «loi élémentaire d'éloignement » suivante :

Pour des stations sifuées à la même altitude mais à des distances différentes de la mer, les précipitations décroissent suivant une fonction exponentielle de l'éloignement.

\section{Lor GÉNÉRALE.}

a) La combinaison de ces lois élémentaires permet d'expliquer l'allure parabolique que traduisent la plupart des formules représentant la loi d'augmentation de la pluviosité avec l'altitude.

Lorsqu'on s'élève en effet sur le flanc d'une

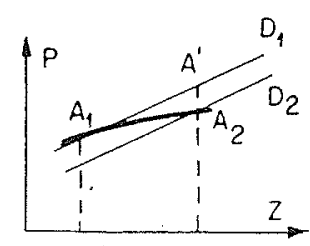

Frg. 17.

montagne exposée aux vents pluvieux, on s'éloigne en même temps de la mer. L'accroissement d'altitude à distance constante fait passer le point figuralif de $A_{1}$ à $A^{\prime}$, l'éloignement de la mer le ramène de $A^{\prime}\left(\right.$ sur $\left.D_{1}\right)$ à $A_{2}$ (sur $D_{2}$ ). I.a résultante des deux «mouvements» donne la courbe d'allure parabolique $A_{1} A_{2}$ qui peut être traduite par la formule suivante -.. à ajouter à toutes ses devancières:

$$
\mathrm{P}=\lambda e^{-\mu \mathrm{D}^{2}}+\mathrm{KZ}
$$

La distance D à la mer d'une station située sur un versant de pente a peut être exprimée en fonction de $\alpha$, de la distance $D_{0}$ (de la base du versant à la mer) et de l'altitude $Z$. On a :

$$
\mathrm{D}=\mathrm{D}_{0}+\left(\mathrm{Z}-\mathrm{Z}_{0}\right) \operatorname{cotg} \alpha
$$

D'où pour P une expression de la forme :

$$
\mathrm{P}=\mathrm{K} Z+\mathrm{K}^{\prime} e^{-(\mathrm{a} x+\mathrm{b}))^{2}}
$$

b) Généralisation. - Loi du maximum en montagne.

La loi mise sous cette forme reste encore «élémentaire» en ce sens qu'elle n'est valable que pour le cas où le vent pluvieux vient d'une direction unique. Supposons maintenant que les pluies arrivent de deux directions principales, les premières dans la proportion $\mathrm{m} \%$, les autres dans la proportion $(100-\mathrm{m}) \%$. La précipitation totale en un point de cote $Z$ sera donnée par une relation telle que :

$$
\mathrm{P}=\mathrm{A} e^{-(\alpha \mathrm{\alpha}+\beta)^{2}}+\mathrm{A}^{\prime} e^{-\left(\alpha^{\prime} z+\beta^{\prime}\right) 2}+\mathrm{BZ}
$$

Il ne peut être question de faire ici la discussion complète de cette équation. Je me bornerai

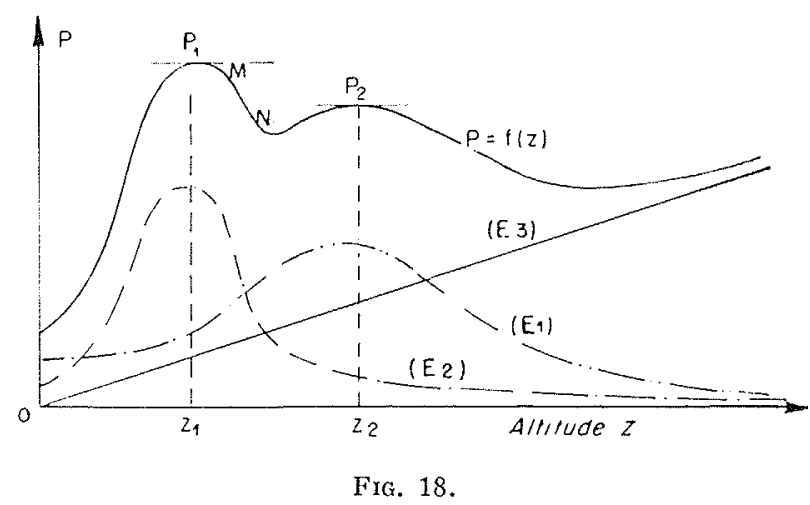

à signaler que si l'on recherche sur cette courbc les points à tangente horizontale, c'est-à-dire les valeurs de $Z$ annulant la dérivée de la fonction $P(Z)$, on peut trouver suivant les valeurs des constantes $\mathrm{A}, \alpha, \ldots \mathrm{B}$, de zéro à quatre solutions réelles. A chacune correspond un maximum ou un minimum de la valeur de $P$. Or $Z$ est une 
grandeur physique, l'altitude, qui varie de 0 a $Z_{s}$, altitude du sommet de la montagne. Parmi les solutions réelles de l'équation $P^{\prime}(Z)=0$ seules seront donc à retenir celles gui peuvent se trouver dans l'intervalle $0--Z_{s}$. La figure cicontre represente un exemple de courbe $\mathrm{P}=1$ (Z) résultante des deux courbes élémentaires $\mathrm{F}_{1}$ $\mathrm{E}_{2}$ (lois exponentielles d'éloignement) et de la droite $\mathrm{E}_{3}$ (loi linéaire d'altitude).

Cela permet d'expliquer quelques faits expérimentatux, par exemple:

a) L'existence d’un maximum de pluviosité en montagnt. Crest une loi que l'on peut énoncer ainsi : dans certaines conäitions bien définies de situation et d'orientation du massif montagneux, les précipitations croissent jusqu'à un premier maximum $P_{1}$ pour l'altitude $Z_{1}$, puis commencent à décroître. Dans les massif́s élevés il peut exister un second maximum $P_{2}$ pour une altitude $Z_{2}>Z_{1}$. Après un nouveau palier, les précipita- tions doivent se remettre théoriquement à augmenter si l'altitude croit indéfiniment.

Le premier maximum $P_{1}$ correspond à l' $l^{\prime}$ optimum pluvial » en montagne, le second à un optimum secondaïe, à altitude supérieure, et qui a parfois été signalé par quelques auteurs;

b) La décrossance dans certains cas particuliers de la pluviosité avec l'altitude. On en trouve un exemple très net sur la côte est de Madagascar soumise alternativement à l'intluence des venls alizés de l'E. - S.-F. et de la mousson d'O. N.-O. La place me manque pour développer un peu plus longuement cet exemple: je me bornerai à indiquer que la côte est de l'île recueille environ $3 \mathrm{~m}$ de pluie. Dans l'intérieur, à environ $120 \mathrm{~km}$ de la côte et à une altitude de $1.600 \mathrm{~m}$, on n'a plus que $1 \mathrm{~m}$ d'eau. Il est très vraisemblable que l'on se trouve sur une portion de courbe telle que M N.
M. le Président remercie M. Serra de son intéressante communication et remarque que les pluviomètres ne sont pas, en fait, aussi mauvais qu'on pourrait le croiro d'après la première partie de celle-ci.

M. Varlet demande à M. Serra ce qu'il pense de la loi de Mathias qu'il a vu imprimée dans la Houille Blanclee et qui, contenant un terme négatif, domnerait a la pluie une allure décroissante avec l'altitude.

M. Senha précise que la loi de Mathias citée par Lugbon et critiquée par Wassov (Allemand) ne doit représenter qu'un «morceau » de la loi générale par suite d'une interprétation plus ou moins correcte des mesures.

Elle comprend un terme en $Z$ croissant plus vite au départ que le terme négatif en $(Z / 100)$ zet ne commence à décroître que pour des valeurs très élevées de $Z$ (théoriquement de l'ordre de $8.000 \mathrm{~m}$ ). Elle ne rend donc pas compte du maximum effectivement observé pour des altitudes bien inférieures.

Sur la demande de $M$. Ie Président M. Senna indique que la loi de variation de la pluie en fonction de l'altitude et de la distance à la mer n'a pas fait l'objet d'études précises à l'étranger, et que les courbes qu'il a présentées résultent de chiffres donnés par le Service Météorologique.

M. Ie Président pense qu'il serait intéressant d'étudiel la variation de la pluie en Bretagne et la répartition des pluviomètres, dont certains doivent être placés sur les versants abrités afin davoir une moyenne plus exacte que les seules indications des apparils exposés à la pluie.

Répondant à une question de M. Varder sur le nombre de pluviomètres qui serait techniquement nécessaire par $\mathrm{km}^{2}$, M. SEnRa indique que ce gros probleme fait actuellement l'objet de plusieurs études, parmi lesquelles les recherches du Service des Etudes et Recherches Hydrauliques d'E.D.F. sur le terrain d'expériences d'Alrance (près le chantier du Pouget) dans l'Aveyron : sur ce terl'ain les données de 16 pluviomètres placés à $30 \mathrm{~m}$ l'un de l'autre dans un carré de $100 \mathrm{~m} \times 100 \mathrm{~m}$ ont montré des écarts allant jusqu'à 20 ou $25 \%$ pour certaines averses; par conséquent la proportion idéale de pluviomètres serait nécessairement supérieure à celle de ces expériences.

En vue de la déterminer, M. Serra a resserré quelques-uns de ces pluviometres jusqu'à un espacement de $10 \mathrm{~m}$ et envisage d'aller jusqu'aux pluviomètres jointifs. En Amérique existe un champ de 55 pluviomètres situés à $1,5 \mathrm{~km}$ l'un de l'autre, où on a trouvé des écarts du même ordre.

M. Varles souligne l'extreme réduction du nombre effectif de pluviomètres; M. Coutagne considère comme particulièrement liches certaines régions de Savoie qui comportent 1 pluviomètre tous les $30 \mathrm{~km}^{2}$.

M. le Président Gariel remarque que la répartition des pluviomètres est liée à la notion de rafales, comme cela se voit dans les observations sur l'énergie du vent.

M. Serra ajoute que les erreurs provenant de l'irregularité directionnelle de la pluie et de son intensité se réduisent par compensation lorsqu'on totalise les données pluviométriques d'une période assez longue : mois on annee.

Sur la demande de M. Fontane, M. Serna indique que l'interprétation des données sur un bassin rersant est soumise dans une certaine mesure à l'évaluation personnelle.

M. Pardí estime que la communication de M. Serra est une excellente mise au point. La mesure des précipitations en montagne est toujours difficile, qu'elle se 
fasse dans des totalisateurs Mougin, forcément sujets à crreur, ou au moyen de pluviomètres dont le nombre est généralement insuffisant et la répartition imparfaite. Dans un bassin d'expériences, celui de la Baye de Montreux, M. Lutscin a installé 30 ou 40 totalisateurs sur 20 ou $25 \mathrm{~km}^{2}$, le long des pentes où les précipitations amnelles varient de 500 à $800 \mathrm{~mm}$ au pied jusqu'ì $2 \mathrm{~m}$ et $2,25 \mathrm{~m}$ en haut; mais, comme $\mathrm{M}$. Covtagne le dit, le meilleur pluviometre est le bassin versant de la rivière qui agit comme un intégrateur idéal. D'autre part, les lois de variation de la pluviosité en fonction de l'altitude, avec maximum entre 2.500 et $3.000 \mathrm{~m}$, étudiées par M. BÉNíveNT dans un hassin d'expériences et appliquées à quelques bassins alpins considérés par M. Pandé dans sa thèse sur le Thone, ont confirmé à très peu de chose près les coefficients d'écoulement donnés par ce dernier ou vice-versa: mais dans cette confirmation romarquable, le hasard a eu certainement sa part, car lesdits coefficients, d'après M. PAnDÉ lui-mème, n'étaient que des approximations grossières.

Répondant à une question de M. le Président, M. Pandé signale d'autre part que la loi des deux maxima a été vérifiée au-dessus de Modane sur la montagne de la Belle-Plinier, ce qui s'explique paree qu'en moyenne on a des précipitations venant de l'ouest et d'autres venant de l'est.

M. Christian Brau est assez heureux de constater que les données pluviométriques sont, malgré la médiocrité des appareils et l'imperfection de leurs lectures, souvent. subjectives, suffisamment utiles dans la pratique, à condition d'interpréter d'après leurs valeurs relatives le sens de la variation; à tel point que ces données, intégrées dans la méthode BAcHer, permettent de faire des annonces de crues du Grand-Morin sur Paris (lesquelles peuvent faire varier le niveau de la Seine de 50 a $80 \mathrm{~cm}$ ) 3 jours à l'avance et a 5 ou $6 \mathrm{~cm}$ près, en tenant compte. en outre, des prévisions météorologiques de quantité de pluie sur le bassin, car les eaux du Grand-Morin arrivent en 24 ho sur Paris.

M. Ie Président conclut en faveur de la multiplication des pluviomètres.

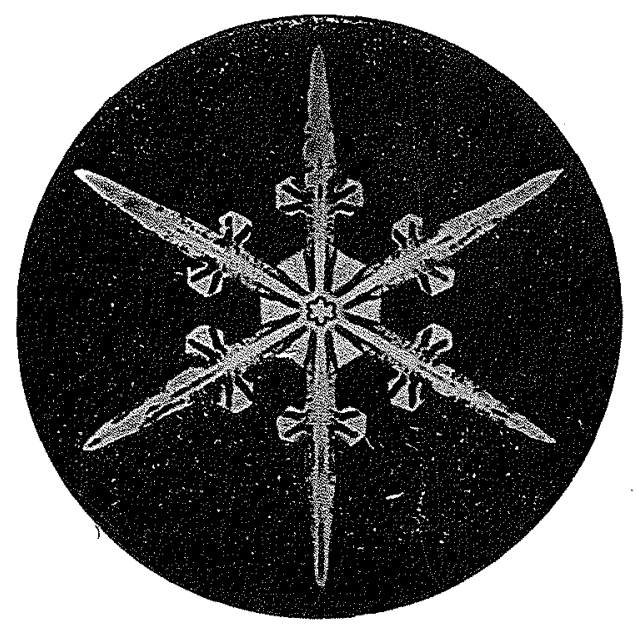

